\section{CP 01}

\section{Comment construire un curriculum interprofessionnel?}

\author{
Patricia PICCHIOTTINO ${ }^{1}$, Elisabeth VAN GESSEL ${ }^{2}$ \\ ${ }^{1}$ Haute École de santé, Centre médical universitaire, avenue de \\ Champel 7, 1206 Genève, Suisse \\ ${ }^{2}$ Faculté de médecine, Centre médical universitaire, avenue de \\ Champel 7, 1206 Genève, Suisse
}

Si l'on se réfère au rapport du Lancet par Julio Frenk et al. [1], datant déjà de 2010, la formation des professionnels en santé est en inadéquation avec les besoins de la pratique médico-soignante de demain ; cette dernière se dirigera dans nos pays industrialisés vers la prise en charge d'une population âgée et polymorbide, dont les soins seront essentiellement communautaires et domiciliaires et leur efficacité nécessitera d'acquérir obligatoirement des compétences collaboratives qui font totalement défaut dans les curriculums actuels. Outre l'orientation future des pratiques, la persistance d'une pédagogie en «silo» et le manque de résultats dans la sécurité des soins, rendent impératives le développement des compétences nécessaires aux pratiques collaboratives [2]. Enfin, il semble indispensable de s'attaquer aux représentations, valeurs et croyances au sein même des professionnels de santé $[3,4]$, qui doivent être abordées précocement dans la formation.

Afin de mener une réflexion pertinente, il s'agit de se référer à la littérature pour comprendre, d'une part les facteurs qui peuvent favoriser la mise en place de ce type de formation, et d'autre part l'impact que cette formation peut avoir. La dernière revue systématique de Reeves et al. [5] a colligé 46 études. Ce dernier a utilisé le modèle d'analyse de Biggs pour juger de la pertinence des dispositifs interprofessionnels, structuré en trois catégories : le contexte (Presage), les processus de mise en œuvre (Process) et leur impact (Outcomes). Si les auteurs ont pu confirmer l'importance du contexte, comme le soutien institutionnel, la motivation des apprenants et des éducateurs à embrasser ces nouvelles formations, ils relèvent surtout dans les processus, l'utilisation d'outils pédagogiques tels que la simulation et particulièrement les dispositifs pratiquant la facilitation et le feed-back. En termes d'impact, la majorité des formations prégraduées ont montré une efficacité pédagogique comme la réaction positive des apprenants, la modification de leurs perceptions et attitudes, ainsi que l'acquisition de connaissances sur la pratique collaborative. Toutefois, ce ne sont que les formations interprofessionnelles au niveau postgradué ou continu qui ont montré quelques effets positifs sur les changements de comportement des professionnels voire des changements dans les pratiques ou le devenir de patients. Faut-il toutefois négliger ce développement et attendre des preuves tangibles? À Genève, nous avons choisi d'implémenter une formation interprofessionnelle précoce sur la base de l'objectif suivant : amener les futurs professionnels de santé à bâtir et comprendre leur identité professionnelle propre tout en leur donnant une compréhension du rôle des autres dans l'équipe de soins. Sur la base du référentiel canadien du Canadian interprofessional health collaborative (CIHC) [6], les domaines de compétences travaillés sont notamment : la communication entre professionnels et avec le patient et ses proches, l'entraînement d'outils nécessaires au travail d'équipe (communication structurée, transmissions, conscience situationnelle, organisation d'une équipe de soins) et l'identification des rôles et responsabilités des différents professionnels. La structure pédagogique s'articule sur trois modules perlés sur l'ensemble de la formation de tous les professionnels de santé genevois. Inspiré des bonnes pratiques décrites par Bridges et al. [7], les modules comprennent un programme didactique, des ateliers de simulations interprofessionnelles et une gestion de projets communs, visant la qualité et la sécurité des soins dans la communauté.

L'évaluation qualitative effectuée lors de la mise en place progressive de ces trois modules a montré chez plus de $80 \%$ des étudiants une prise de conscience de la responsabilité de chacun dans le travail d'équipe, la perception de l'importance des objectifs d'équipe par rapport aux objectifs professionnels individuels (responsabilité collective) et enfin la nécessité de faire valoir sa préoccupation concernant la sécurité du patient (posture dans l'équipe).

\section{Références}

[1] Frenk J, Chen L, Bhutta ZA, Cohen J, Crisp N, Evans T, et al. Health professionals for a new century: transforming education to strengthen health systems in an interdependent world. Lancet 2010;376:1923-58

[2] Thistlewaite J, Jackson A, Moran M. Interprofessional collaborative practice: a deconstruction. J Interprof Care 2013;27:50-6

[3] Muller-Juge V, Cullati S, Blondon KS, Hudelson P, Maître F, et al. Interprofessional collaboration on an internal medicine ward: role perceptions and expectations among nurses and residents. PLoS ONE 2013;8(2):e57570. DOI: 10.1371/journal.pone.0057570

[4] Muller-Juge V, Cullati S, Blondon KS, Hudelson P, Maître F, et al. Interprofessional collaboration between residents and nurses in general internal medicine: a qualitative study on behaviours enhancing teamwork quality. PLoS ONE 2014;9(4):e96160. DOI: 10.1371 journal.pone.0096160

[5] Reeves S, Fletcher S, Barr H, Birch I et al. A BEME systematic review of the effects of interprofessional education: BEME Guide no 39. Med Teach 2016;38:656-68.

[6] The Canadian interprofessional health collaborative (CIHC). A national interprofessional competency framework. 2010 [On-line] Disponible sur: www.cihc.ca

[7] Bridges DR, Davidson RA, Odegard PS, Maki IV, Tomkowiak J. Interprofessional collaboration: three best practice models of interprofessional education. Medical Education Online 2011;16:6035. DOI: $10.3402 /$ meo.v16i0.6035

\section{CP 02}

\section{La place de la réalité virtuelle dans les formations en santé}

\author{
Valérie CAMPILLO-PAQUET $T^{1,2}$
}

1 Chargée de mission numérique pour l'enseignement, Aix-Marseille Université, France

${ }^{2}$ Chercheur au laboratoire d'économie et de sociologie du travail (UMR 7317), France 
Cette communication aborde l'usage pédagogique - actuel et à venir- des dispositifs numériques immersifs, tels que la réalité virtuelle (RV) et la réalité augmentée (RA), dans les formations en santé. En effet, le secteur médical a toujours été parmi les pionniers en matière d'outils de simulation, autant dans le champ pédagogique que professionnel et l'essor du numérique ainsi que les avancées technologiques et informatiques offrent à présent des outils de simulation virtuelle, hautement réalistes, potentiellement intéressants dans le cadre des apprentissages.

La première partie présente et précise les dispositifs numériques de type RV et RA, en particulier les technologies utilisées, les différences et les champs d'utilisation actuels. La suite de cette présentation vise à répondre à trois questions relatives à l'usage de ces dispositifs dans les formations initiales et continues en santé.

Quel sont les actuelles avancées en matière de réalité virtuelle (RV) et de réalité augmentée (RA) pour la formation en santé ? Cette partie effectue un tour d'horizon des principaux cadres d'usage dans le domaine de la formation (initiale et continue) en santé : De la «cyberanatomie », permettant d'explorer le corps humain de manière réaliste et commentée, à la simulation d'actes chirurgicaux «in virtuo», la panoplie des usages pédagogiques permet aux étudiants et stagiaires de FC de s'entraîner dans un environnement réaliste et non invasif (pose de diagnostic, gestes médicaux, actes chirurgicaux, etc.).

En quoi les techniques de RV et RA peuvent-elles améliorer la formation en santé ? Plusieurs avantages sont présentés dans cette partie, tant du point de vue pédagogique (modalité d'apprentissage par la pratique, essais-erreurs, développement de compétences complémentaires : communication, coordination d'équipes, gestion des risques...), que du point de vue de l'accès aux connaissances, ou tout au moins aux données. Les représentations 3D immersives, voire holographiques, offrent de véritables bases de données anatomiques, pathologiques, pouvant être explorées « de l'intérieur », du général au détail. Ces dispositifs permettent en outre un apprentissage à distance, atténuent les contraintes géographiques et matérielles (accès aux laboratoires, aux salles physiques de simulation, observation en salle d'opération...).

Quelles sont les limites actuelles de ces dispositifs dans le paysage éducatif en santé ? L'intérêt de la RV et de la RA pour la formation en santé se heurte néanmoins à de nombreux freins, principalement techniques et financiers. D'autres facteurs, tels que l'appropriation de ces dispositifs par les usagers (praticiens, enseignants et apprenants), la validation des cas proposés et surtout la jeunesse de ces nouveaux objets numériques limitent encore leur essor.

\section{$\mathrm{CP} 03$}

\section{Comment favoriser le développement du raisonnement clinique grâce aux environnements numériques d'apprentissage?}

\section{Joseph-Omer DYER}

École de réadaptation, Faculté de médecine, Université de Montréal, Montréal (QC) Canada
Introduction: À l'heure actuelle, les technologies de l'information offrent des possibilités énormes en ce qui a trait à l'enseignement et à l'apprentissage dans une multitude de domaines de savoir. Ces technologies sont très diversifiées. Elles incluent, entre autres, les réseaux sociaux, les applications mobiles, et les environnements numériques d'apprentissage. Le terme e-learning fait référence à l'utilisation de ces technologies numériques de l'information dans le but de favoriser l'apprentissage. L'utilisation, dans un cadre pédagogique, de sources d'information déjà disponibles sur Internet (ex. : sites spécialisés, bases de données, vidéos, etc.) est la forme la plus simple d'e-learning. Cependant, l'e-learning peut impliquer des ressources plus complexes dont des environnements numériques d'apprentissage (ENA) spécifiquement conçus pour favoriser des apprentissages ciblés. Ces ENA peuvent intégrer diverses ressources dont des images, du son, des vidéos, des cas de patients virtuels, de la modélisation numérique de systèmes ou processus, et des didacticiels en ligne. Bien que ces différentes ressources aient un fort potentiel pour permettre l'apprentissage, il n'est pas clair comment les ENA doivent être conçus pour optimiser le développement d'une compétence complexe telle que le raisonnement clinique des professionnels de la santé.

Problématique et objectif : L'apprentissage du raisonnement clinique à l'aide des ENA pose plusieurs défis. Un premier défi est le fait que le raisonnement clinique utilise des savoirs tacites acquis avec l'expérience clinique, et que de tels savoirs peuvent être difficiles à transposer en ENA. De plus, l'ENA doit être conçu, ou doit pouvoir s'adapter en fonction du niveau de compétence de l'apprenant (ex.: novice vs. avancé). Cette présentation vise à donner aux formateurs des professions de la santé des ressources pour faire face à ces défis. L'objectif principal de cette présentation est de décrire le cadre théorique et les principes sur lesquels les enseignants peuvent s'appuyer pour développer des ENA qui facilitent l'apprentissage du raisonnement clinique. Ce cadre théorique inclut la théorie de la charge cognitive, la théorie cognitive de l'apprentissage multimédia et l'effet d'apprentissage au moyen des «worked examples» (exemples résolus). Un tel cadre présente des principes utiles à l'élaboration des ENA qui sont basés sur des preuves.

Théorie de la charge cognitive : Cette théorie stipule que la mémoire de travail humaine a une capacité limitée et que la surcharge de cette mémoire nuit à l'apprentissage [1]. À cet égard, il importe donc que les ENA ne produisent pas de surcharge cognitive chez les apprenants. Les ENA devraient limiter l'information présentée à celle qui est véritablement utile à l'apprentissage et éviter de présenter toute information redondante ou non pertinente. L'échelle subjective de perception de l'effort mental investi pendant une activité permet une estimation valide de la charge cognitive au cours de cette activité [2]. Cette échelle est simple à mettre en place et à utiliser. Elle permet, entre autres, de comparer l'effort mental investi entre différentes activités en ENA et de mettre en évidence celles dans lesquelles les apprenants sont plus à risque de surcharge cognitive. Les mesures effectuées avec cette échelle peuvent être associées aux résultats des tests d'évaluation des apprentissages pour estimer l'efficience cognitive des activités. Ainsi, les activités sont considérées 
comme étant efficientes en termes de processus d'apprentissage lorsque l'effort mental investi par les apprenants est faible pendant l'activité d'apprentissage et que ces apprenants démontrent des apprentissages significatifs post-activité. Par ailleurs, les activités sont considérées comme étant efficientes en termes de résultats d'apprentissage lorsque l'effort mental investi par les apprenants est faible pendant les tests d'évaluation des apprentissages et que ces apprenants démontrent des apprentissages significatifs [3].

Théorie cognitive de l'apprentissage multimédia: Cette théorie présente des principes pour développer les ENA de manière à optimiser les capacités de la mémoire de travail des apprenants [4]. Ainsi, cette théorie stipule, entre autres, que les capacités de la mémoire de travail des apprenants est accrue lorsque les activités d'apprentissage utilisent simultanément et de manière coordonnée les modalités sensorielles visuelle et auditive. Par exemple, un ENA qui présente l'information sous la forme d'une vidéo qui combine à la fois les images et la narration de manière complémentaire permettra à l'apprenant de plus facilement traiter l'information à apprendre par rapport à un ENA présentant la même information sous la forme d'images, de textes ou de narration seulement [5].

Effet d'apprentissage au moyen des «worked examples» (exemples résolus): Les principes liés à cet effet permettent de planifier les activités d'enseignement pour optimiser les apprentissages chez des apprenants novices. Dans ce contexte, les novices sont des apprenants qui ont peu ou pas de connaissances dans le domaine d'apprentissage ciblé. Chez ces apprenants novices, les données de la recherche montrent que l'apprentissage à l'aide d'exemples présentant la démarche complète de résolution d'un problème est plus efficace que l'apprentissage à l'aide d'activités basées sur la résolution de problèmes [6]. De tels exemples résolus ont été utilisés pour promouvoir l'apprentissage du raisonnement clinique chez des apprenants des professions de la santé [7,8]. Ces exemples résolus peuvent être présentés en ENA et prendre la forme de cas cliniques à partir desquels est présentée la démarche complète qui mène au diagnostic ou à la sélection de l'intervention la plus appropriée en regard du problème clinique du patient et du contexte $[9,10]$. Des recherches sont menées actuellement pour mieux comprendre comment les exemples de cas cliniques résolus pour développer le raisonnement clinique qui sont présentés en ENA peuvent être améliorés à l'aide d'activités réflexives (ex. : auto-explication), ou des activités d'organisation des connaissances (ex.: cartes conceptuelles) [10].

Conclusion : Il existe des principes théoriques basés sur des données de la recherche qui peuvent guider les formateurs dans l'élaboration des activités de développement du raisonnement clinique dans les ENA. Ces ENA doivent être élaborés de manière à éviter la surcharge cognitive et à utiliser les possibilités du multimédia pour optimiser les processus cognitifs des apprenants. Chez les apprenants novices, les ENA devraient inclure des exemples de cas cliniques présentant la démarche complète de résolution de problème pour trouver le diagnostic ou sélectionner l'intervention optimale. Les études futures permettront de mieux comprendre l'impact des activités réflexives et d'organisation des connaissances sur les effets d'apprentissage des ENA.

\section{Références}

[1] Sweller J. Cognitive load theory, learning difficulty, and instructional design. Learning and Instruction 1994;4(4):295-312.

[2] Paas FG, Van Merrienboer JJ,. Adam JJ. Measurement of cognitive load in instructional research. Percept Mot Skills 1994;79(1 Pt 2):419-430.

[3] Van Gog T, Paas F. Instructional efficiency: revisiting the original construct in educational research. Educational Psychologist 2008;43 (1):16-26.

[4] Mayer RE. Applying the science of learning: evidence-based principles for the design of multimedia instruction. Am Psychol 2008;63(8):760-769.

[5] Mayer RE, Hegarty M, Mayer S, Campbell J. When static media promote active learning: annotated illustrations versus narrated animations in multimedia instruction. J Exp Psychol Appl 2005;11 (4):256-265.

[6] Sweller J. The worked example effect and human cognition. Learning and instruction 2006;2(16):165-169.

[7] Kopp V, Stark R, Kuhne-Eversmann L, Fischer MR.Do worked examples foster medical students' diagnostic knowledge of hyperthyroidism? Med Educ 2009;43(12):1210-7.

[8] Stark R, Kopp V, Fischer MR. Case-based learning with worked examples in complex domains: Two experimental studies in undergraduate medical education. Learning and instruction 2011;21(1):22-33.

[9] Kopp V, Stark R, Fischer MR. Fostering diagnostic knowledge through computer-supported, case-based worked examples: effects of erroneous examples and feedback. Med Educ 2008;42(8):823-829.

[10] Dyer JO, Hudon A, Montpetit-Tourangeau K, Charlin B, Mamede B, van Gog T. Example-based learning: comparing the effects of additionally providing three different integrative learning activities on physiotherapy intervention knowledge. BMC Med Educ 2015;5:37.

\section{CP 04 \\ Comment innover dans ses pratiques d'enseignement?}

\section{Marilou BELISLE}

Faculté d'éducation, Université de Sherbrooke, Sherbrooke (QC), Canada

D'où provient l'idée d'innover dans ses pratiques d'enseignement? Pour qui et pourquoi innove-t-on? Ces questions banales sont pourtant déterminantes pour les personnes qui innovent. Si l'innovation pédagogique se conçoit comme une activité délibérée qui a pour but d' « améliorer substantiellement les apprentissages des étudiants en situation d'interaction et d'interactivité» [1], quelle démarche peut-on alors entreprendre pour innover de manière rigoureuse, éclairée et bénéfique pour l'apprentissage?

En réponse à cette première question, nous proposons une démarche d'innovation pédagogique qui tient compte des trois temps de l'innovation (conception, implantation et évaluation) [2] et qui s'appuie sur un modèle de développement de l'expertise sur 
l'enseignement et l'apprentissage, le SOTL - Scholarship of Teaching and Learning [3]. Cette démarche se décline en six phases : 1) l'analyse de sa pratique et de son contexte d'enseignement en vue d'identifier un besoin ou de résoudre un problème lié à l'apprentissage des étudiants ; 2) l'appropriation de connaissances permettant d'éclairer sa réflexion et d'informer sa pratique actuelle et future; 3) la conception du changement à mettre en œuvre; 4) l'implantation du changement; 5) l'évaluation des retombées du changement sur l'apprentissage et l'enseignement et : 6) la diffusion des retombées auprès de la communauté professionnelle ou scientifique [4]. Ces phases sont imbriquées les unes aux autres et chacune d'elles peut constituer une porte d'entrée pour enclencher un processus d'innovation dans ses pratiques d'enseignement.

La seconde question met l'accent sur la phase de conception du changement : comment scénariser le changement à mettre en œuvre? Bien que plusieurs modèles de planification ou de design pédagogique reconnus dans la littérature permettent de soutenir la scénarisation des activités de formation, trois principes clés seront abordés pour baliser les contours de toute démarche d'innovation et favoriser un apprentissage durable et en profondeur: l'alignement pédagogique [5], l'engagement des étudiants [6], la transparence, et la rétroaction [7].

La troisième question concerne la phase de l'évaluation: quelles données colliger pour documenter les retombées d'une innovation pédagogique? La satisfaction de l'expérience vécue, les perceptions à l'égard de l'activité de formation, les apprentissages effectifs sont autant de données utiles pour renseigner ses pratiques. Toutefois, la réponse à une telle question sera tributaire des apprentissages visés par l'innovation, des connaissances méthodologiques de la personne qui innove, ainsi que des ressources disponibles dans son environnement. Des exemples illustreront la démarche SOTL proposée pour innover dans ses pratiques d'enseignement de manière rigoureuse, éclairée et bénéfique pour l'apprentissage.

\section{Références}

[1] Béchard J-P, Pelletier P. Les universités traditionnelles : à l'heure des innovations pédagogiques? Gestion 2004;29(1):48-55

[2] Bédard D, Béchard J-P. (dir.). Innover dans l'enseignement supérieur. Paris : Presses Universitaires de France, 2009

[3] O'Brien M. Navigating the SoTL landscape: a compass, map and some tools for getting started. Int J Scholarsh Teach Learn 2008;2(2): 15 .

[4] Bélisle M, Lison C, Bédard D. 2016 Accompagner le Scholarship of Teaching and Learning. in Comment développer le conseil pédagogique dans l'enseignement supérieur? Cadres de référence, outils d'analyse et de développement, A Daele, E Sylvestre, Editors. Bruxelles: De Boeck, p. 75-90.

[5] Biggs J. Enhancing teaching through constructive alignment. High Educ 1996;32(3):347-364.

[6] Viau R. Savoir motiver les étudiants. in Se former à la pédagogie de l'enseignement supérieur, L Ménard, L St-Pierre, Editors. Montréal: AQPC, 2014:235-254.

[7] Boud D, Molloy E. Rethinking models of feedback for learning: the challenge of design. Assessment \& Evaluation in Higher Education 2012;38(6):698-712.

\section{CP 05}

\section{Peut-on évaluer les enseignants?}

\author{
Valérie Dory \\ Université Mc Gill, Montréal (QC), Canada \\ Contact : valerie.dory@mcgill.ca
}

L'évaluation des enseignants du supérieur est apparue en Amérique du Nord il y a près d'un siècle et son utilisation s'y est répandue dans les années 1960-1970, pour devenir aujourd'hui courante dans de nombreux pays [1]. L'outil le plus fréquemment utilisé est le questionnaire aux étudiants, typiquement utilisé en fin de session, mais parfois aussi en deux temps [2]. Il existe d'autres manières de recueillir les perceptions des étudiants telles que le sondage rapide ou les groupes focaux [3].

Au-delà du recueil des perceptions des étudiants, certains préconisent l'évaluation des enseignants par des pairs ou des pédagogues. Ces évaluations peuvent se baser sur une ou des observations de l'enseignant en situation de cours mais également comprendre une analyse documentaire et des entretiens avec l'enseignant [4]. Dans le domaine des sciences de la santé, des examens d'enseignement objectifs structurés ont été déployés [5]. De plus, l'utilisation des résultats des étudiants en fin de cours, dans les cours suivants, ou encore dans des examens standardisés peuvent être utilisés comme indicateurs de l'efficacité de l'enseignement dispensé [4]. Enfin, les portfolios sont devenus de plus en plus courants dans le supérieur [2]. Les études visant à valider les questionnaires aux étudiants foisonnent, notamment l'examen de biais potentiels [1]. En réalité, les biais (liés au sexe, à l'appartenance à une minorité visible, à la personnalité de l'enseignant), s'ils existent, ont un impact faible sur les évaluations [1]. Les scores issus des évaluations par les étudiants sont corrélés à d'autres mesures (auto-évaluation, observations) [1]. En revanche, le lien entre ces scores et les apprentissages est un sujet très controversé et les méta-analyses récentes semblent indiquer une absence de lien ou du moins un lien ténu [6]. Par ailleurs, les conceptions des étudiants et des enseignants sur ce qu'est un bon enseignement divergent en partie [1] et le recours habituel aux questionnaires aux étudiants comme unique source de données révèle une conception réductrice de l'enseignement et de l'apprentissage. La littérature révèle une problématique cruciale dans ces processus évaluatifs, à savoir que leur validité dépend de l'utilisation des évaluations et non pas uniquement des propriétés des outils de mesure. Leur utilisation est en réalité plurielle. Les buts poursuivis par ces processus également. Ils ont en effet une fonction formative basée principalement sur la rétroaction fournie à l'enseignant. Ils ont également des fonctions sommatives au niveau de l'enseignant (recrutement, promotion, statut permanent), mais aussi au niveau institutionnel, avec dans certains pays un lien avec le financement de l'institution [1]. Ces buts émergent de visions philosophiques différentes, en particulier une vision académique professionnelle, dans laquelle on accorde une confiance aux académiques, notamment quant à leur jugement professionnel et à leur engagement dans un processus de formation continue, avec de l'autre une vision managériale de la gestion académique où la confiance est remplacée 
par l'audit, avec des valeurs prônées telles que la transparence et l'imputabilité [1,7]. Si le souhait de valoriser l'enseignement au même titre que la recherche dans les universités est bien intentionné, la mise en œuvre managériale de ce processus risque de créer des effets pervers, bien connus dans le domaine de l'évaluation des apprenants-à savoir les comportements stratégiques des enseignants - sans compter le risque de démotivation $[7,8,9]$. La manière dont les processus sont mis en place-diversité des sources de données, qualité de leur interprétation, incitants, type de rétroactionaura donc un impact sur leurs effets tant sur les parties prenantes qu'in fine sur la qualité de l'enseignement dispensé [9-12].

Les processus d'évaluation sont en réalité des systèmes complexes dans lesquels des agents poursuivant des objectifs différents s'influencent mutuellement. Toutefois, une réflexion s'impose sur la possibilité même de poursuivre explicitement plusieurs buts et il semblerait opportun de les hiérarchiser, en fonction d'une vision cohérente du monde académique. Diverses pistes tenant compte de choix clairs s'ouvrent à notre communauté. Ces pistes se fondent sur ce que nous savons actuellement du processus d'apprentissage tant chez les étudiants que chez les enseignants. L'apprentissage n'est pas une ingurgitation de contenus transmis. Il est une co-construction de savoirs. L'enseignement n'est pas une performance théâtrale, il est une pratique experte complexe reposant sur des savoirs disciplinaires et pédagogiques déployés de manière adaptative. Les processus évaluatifs visant à soutenir l'enseignement et l'apprentissage doivent être alignés avec ces conceptions $[2,8,13]$.

\section{Références}

[1] Spooren P, Brockx B, Mortelmans D. On the validity of student evaluation of teaching: The state of the art. Rev Educ Res 2013;83 (4):598-642.
[2] Saroyan A, Amundsen C. Evaluating university teaching: time to take stock. Assess Eval High Educ 2001;26(4):341-53.

[3] Huxham M, Laybourn P, Cairncross S, et al. Collecting student feedback: a comparison of questionnaire and other methods. Assess Eval High Educ 2008;33(6):675-86.

[4] Berk RA, Naumann PL, Appling SE. Beyond student ratings: peer observation of classroom and clinical teaching. Int J Nurs Educ Scholarsh 2004;1:10.

[5] Trowbridge RL, Snydman LK, Skolfield J, Hafler J, Bing-You RG. A systematic review of the use and effectiveness of the objective structured teaching encounter. Med Teach 2011;33(11):893-903.

[6] Uttl B, White CA, Gonzalez DW. Meta-analysis of faculty's teaching effectiveness: Student evaluation of teaching ratings and student learning are not related. Stud Educ Eval 2017;54:22-42.

[7] Valsan C, Sproule R. The invisible hands behind the student evaluation of teaching: The rise of the new managerial elite in the governance of higher education. J Econ Issues 2008;42(4):939-58.

[8] Trigwell K. Judging university teaching. Int J Acad Dev 2001;6 (1):65-73.

[9] Arthur L. From performativity to professionalism: lecturers' responses to student feedback. Teach High Educ 2009;14(4):441-54. [10] Darwin S. What contemporary work are student ratings actually doing in higher education? Stud Educ Eval 2017;54:13-21.

[11] Penny AR, Coe R. Effectiveness of consultation on student ratings feedback: a meta-analysis. Rev Educ Res 2004;74(2):215-53.

[12] Penny AR. Changing the agenda for research into students' views about university teaching: four shortcomings of SRT research. Teach High Educ 2003;8(3):399-411.

[13] Nygaard C, Belluigi DZ. A proposed methodology for contextualised evaluation in higher education. Assess Eval High Educ 2011;36(6):657-71. 